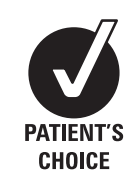

- A supplementary video is published online only at http:// jnnp.bmj.com/content/vol80/ issue2

${ }^{1}$ Centre of Neurology and Hertie Institute for Clinical Brain Research, University of Tübingen, Germany;

${ }^{2}$ Department of Neuroradiology, University of Tübingen, Germany; ${ }^{3}$ Department of Neurosurgery, University of Tübingen, Germany

Correspondence to: Dr R Krüger, Centre of Neurology and Hertie Institute for Clinical Brain Research, Hoppe-SeylerStr 3, 72076 Tübingen,

Germany; rejko.krueger@ uni-tuebingen.de

S Breit and T Wächter contributed equally to this work.

Received 27 January 2008 Revised 7 April 2008 Accepted 16 May 2008

\title{
Effective thalamic deep brain stimulation for neuropathic tremor in a patient with severe demyelinating neuropathy
}

\author{
S Breit,, T Wächter, ${ }^{1}$ L Schöls, ${ }^{1} T$ Gasser, ${ }^{1}$ T Nägele, ${ }^{2}$ D Freudenstein, ${ }^{3}$ R Krüger ${ }^{1}$
}

\begin{abstract}
Postural and action tremor in peripheral neuropathy is characteristic of Roussy-Levy syndrome. A patient with a severe demyelinating neuropathy and disabling neuropathic tremor successfully treated by deep brain stimulation (DBS) is reported. Disease onset was at age 63 years with sensory symptoms and slight action tremor. Within the following 9 years a severe, drug resistant, postural and action tremor developed rendering the patient unable to feed himself. At age 72 years the patient was treated by bilateral DBS of the ventral intermediate thalamic nucleus, with a useful 30\% reduction in tremor. The clinical benefit of the stimulation remained stable over a 1 year postoperative observation period.
\end{abstract}

Neuropathic tremor is defined as tremor that develops in association with peripheral neuropathy when no other neurological condition associated with tremor is encountered. ${ }^{12}$ Peripheral neuropathy with prominent tremor is characteristic of Roussy-Levy syndrome. ${ }^{3}$ It typically presents as action tremor and is observed mainly in patients with chronic inflammatory demyelinating polyneuropathies (CIDP), or hereditary motor and sensory neuropathies (HMSN). As a potential mechanism, a distorted peripheral sensory input is misleading an otherwise intact central network into producing tremor. ${ }^{4}$ Neuropathic tremor responds poorly to medical treatment. Effective surgical treatment by unilateral deep brain stimulation (DBS) of the ventral intermediate thalamic nucleus (Vim) was shown in one patient with neuropathic tremor and IgM paraproteinaemic neuropathy. ${ }^{5}$ Here we report successful bilateral DBS of the Vim in a patient with severe action and postural tremor caused by demyelinating neuropathy, as seen in Roussy-Levy syndrome.

\section{CASE REPORT}

A 72-year-old male patient with late onset neuropathy and neuropathic tremor first noticed distal sensory symptoms of the limbs at age 63 years. Electrophysiological examination revealed severe sensorimotor neuropathy. Analyses of the CSF showed a variable increase in total protein ranging from 61 to $170 \mathrm{mg} / \mathrm{dl}$. Within 1 year the patient developed slight action and postural tremor and painful dysaesthesia. A sural nerve biopsy revealed a severe chronic demyelinating neuropathy with hypertrophy of myelin sheaths and sporadic perivascular inflammatory response in the epineurium. Although the inflammatory signs were sparse, a probatory treatment with corticosteroids (prednisolone $100 \mathrm{mg} /$ day) was initiated under the diagnosis of CIDP. After 1 year of therapy, no clinical benefit was observed. Therefore, and because of the markedly reduced motor conduction velocities without evidence of acute muscular denervation and the uniform slowing of motor conduction velocity in all examined nerves, subsequently the diagnosis of HMSN I was favoured. Nine years after disease onset, motor conduction velocities were markedly decreased as follows: tibial and ulnar nerve $3 \mathrm{~m} / \mathrm{s}$ $(0.3 \mathrm{mV})$ and $4 \mathrm{~m} / \mathrm{s}(0.3 \mathrm{mV})$, respectively, and peroneal and radial nerve $11 \mathrm{~m} / \mathrm{s}$ each $(1.5 \mathrm{mV}$ and $0.4 \mathrm{mV}$, respectively). Tremor analysis by surface EMG recording of antagonistic wrist muscles showed an irregular $2 \mathrm{~Hz}$ tremor. There was no responsiveness to alcohol. Treatment with propranolol, metoprolol, primidone, clonazepam, pramipexole, tiaprid, amantadine, budipine and gabapentin gave no sustained improvement.

Because of therapy resistant tremor, which interfered with most activities of daily living, surgical treatment by DBS of the Vim of the thalamus was considered. The patient underwent bilateral electrode implantation into the Vim using the CRW stereotactic frame (Radionics, Burlington, Massachusetts, USA). CT-MRI fusion followed by landmark based targeting was used to define the anatomical target at the level of the intercommissural line, $5 \mathrm{~mm}$ anterior to the posterior commissure (PC) and $17 \mathrm{~mm}$ from the midline. Electrophysiological exploration of the targeted brain region was performed using three microrecording/macrostimulation electrodes (FHC, Bowdoinham, Maine, USA). A quadripolar electrode (type 3387; Medtronic, Minneapolis, Minnesota, USA) was inserted into each Vim along the trajectory that yielded best results in electrophysiological exploration and connected to an implantable pulse stimulator (Kinetra, Medtronic, Minneapolis, Minnesota, USA).

\section{RESULTS}

Electrode implantation in the Vim was performed without complications. Electrode coordinates at the AC-PC level, corresponding to the most distal contacts, as measured on postoperative MRI, were $5.5 \mathrm{~mm}$ anterior to the $\mathrm{PC}$ and $16 \mathrm{~mm}$ from the midline for the left Vim and $6.5 \mathrm{~mm}$ anterior to the PC and 19 from the midline for the right Vim. Chronic electrical stimulation was performed using a pulse duration of $210 \mu$ s and a frequency of $130 \mathrm{~Hz}$. The best clinical response was obtained with bipolar stimulation at $4.5 \mathrm{~V}$ between the 
most distal contacts in the left $\operatorname{Vim}(0-, 1+)$ and at $3.5 \mathrm{~V}$ between the second distal contacts in the right Vim $(5-, 6+)$. The right hand tremor showed a marked improvement under stimulation whereas the left hand tremor displayed a moderate tremor reduction (see supplementary video online). Overall stimulation resulted in a useful $30 \%$ reduction in tremor (57 to 41 points), as assessed by a performance based test of the Columbia University Assessment of Disability in Essential Tremor (CADET). ${ }^{6}$ Tremor suppression was further improved by increasing voltage, but increased current, even with decreased pulse width ( $\mu$ s), deteriorated gait ataxia. As an adverse effect, dysarthria was observed at high stimulation amplitude under monopolar conditions, resulting in the bipolar stimulation paradigm. Follow-up examination after 1 year revealed a stable tremor response to DBS despite continuous worsening of the underlying neuropathy with accentuation of gait ataxia.

\section{DISCUSSION}

This case report demonstrates for the first time that bilateral DBS of the Vim can be highly effective in the treatment of severe demyelinating neuropathic tremor seen in Roussy-Levy syndrome. A previous case report demonstrated the effectiveness of Vim DBS in neuropathic tremor associated with IgM paraproteinaemic neuropathy. ${ }^{5}$

Regarding the clinical course, electrophysiological characteristics and findings on nerve biopsy, we feel that neuropathy in our patient was most likely due to HMSN and resembled a condition alternatively known as Roussy-Levy syndrome. ${ }^{3}$ The phenotype of clumsiness of gait and hands, absent deep tendon reflexes, decreased vibration sense in the legs and tremor is genetically heterogeneous and can also be observed in patients without a family history. We could not detect common mutations in the PMP22 and connexin 32 gene, which are responsible for CMT1A and CMTX, but this does not preclude a diagnosis of Roussy-Levy syndrome. ${ }^{8}$ The diagnosis is supported by the lack of gammopathy, metabolic or inflammatory causes and the adult onset with markedly reduced motor conduction velocities. Nevertheless, CIDP is an important differential diagnosis and difficult to exclude. However, increased CSF protein levels have been described in up to $50 \%$ of cases of HMSN-I, and coexistent inflammatory signs were described in genotype independent HMSN. ${ }^{10}$ In our case, the lack of improvement under prolonged corticosteroid therapy and the presence of hypertrophic nerve roots without contrast enhancement in MRI is typical of degenerative disease and argues against an inflammatory process. ${ }^{7}$

As the patient's tremor developed in close association with the peripheral neuropathy without any evidence of another neurological disease, there is no doubt about the diagnosis of neuropathic tremor. ${ }^{12}$ The irregular low frequent tremor, in particular, pronounced at distal parts of the upper limbs, is in line with previous descriptions of this entity. ${ }^{11}$ Worsening of tremor on certain tasks and the aymmmetry could also indicate dystonic tremor in this patient. Indeed, concurrent dystonia was reported in some patients with HMSN. ${ }^{12}$ Thus we cannot exclude an accompanying slight dystonic component contributing to the asymmetry in our patient, although examination revealed no definite signs of dystonia.

Although a peripheral origin of neuropathic tremor is assumed, there is strong rationale for considering DBS of the Vim for the treatment of tremor, regardless to its aetiology. Firstly, physiological studies indicate that distorted peripheral inputs reach a central processor (ie, cerebellum) which although intact is misled into producing tremor. ${ }^{4}$ Secondly, activation studies using positron emission tomography and functional MRI showed cerebellar hemisphere hyperactivity similar to that in essential tremor. ${ }^{513} 14$ Thirdly, the Vim is a key relay structure within the spino-cerebello-thalamo-cortico-spinal loops, being involved in the generation of non-parkinsonian tremor of various aetiologies. ${ }^{1}$ Recent studies indicate that targeting stimulation not to the grey matter, but rather to the more caudally located white matter of the subthalamic area, may be more effective in attenuation of the disabling tremor. ${ }^{14}$ This may correspond with the higher effectiveness of the bipolar stimulation of the more distal electrodes in our patient. Moreover, deterioration of gait ataxia on further increase in stimulation current correlates with a potential involvement of cerebellothalamic projections observed in patients with essential tremor and multiple sclerosis tremor. ${ }^{15}$

In conclusion, the present work adds novel evidence that Vim DBS might be an important therapeutic option to consider in neuropathic tremor refractory to pharmacological therapy.

Funding: The work of RK is supported by a grant from the German Research Council (DFG; KR2119/3-1).

Competing interests: None.

Ethics approval: Ethics approval was obtained.

Patient consent: Obtained.

\section{REFERENCES}

1. Deuschl G, Bergman H. Pathophysiology of nonparkinsonian tremors. Mov Disord 2002;17:S41-8.

2. Bain PG. The management of tremor. J Neurol Neurosurg Psychiatry 2002;72:13-9.

3. Roussy G, Levy G. Sept cas d'une maladie familiale particulaire. Rev Neurol 1926;45:427-50.

4. Bain PG, Britton TC, Jenkins $\mathbf{I H}$, et al. Tremor associated with benign IgM paraproteinaemic neuropathy. Brain 1996;119:789-99.

5. Ruzicka E, Jech R, Zarubova K, et al. VIM thalamic stimulation for tremor in a patient with IgM paraproteinaemic demyelinating neuropathy. Mov Disord 2003;18:1192-5.

6. Wendt KJ, Albert SM, Pullman SL, et al. Columbia University Assessment of Disability in Essential Tremor (CADET): methodological issues in essential tremor research. Parkinsonism Rel Disord 2000;6:17-23.

7. Haubrich C, Krings T, Senderek J, et al. Hypertrophic nerve roots in a case of Roussy-Levy syndrome. Neuroradiology 2002;44:933-7.

8. Nicholson GA. The dominantly inherited motor and sensory neuropathies: Clinical and molecular advances. Muscle Nerve 2006;33:589-97.

9. Hagberg B, Lyon G. Pooled European series of hereditary peripheral neuropathies in infancy and childhood. A "correspondence work shop" report of the European Federation of Child Neurology Societies (EFCNS). Neuropediatrics 1981;12:9-17.

10. Ginsberg L, Malik 0, Kenton AR, et al. Coexistent hereditary and inflammatory neuropathy. Brain 2004;127:193-202.

11. Nobile-Orazio E, Meucci N, Baldini L, et al. Long-term prognosis of neuropathy associated with anti-MAG IgM M-proteins and its relationship to immune therapies. Brain 2000;123:710-17.

12. Cardoso $\mathbf{F E}$, Jankovic J. Hereditary motor-sensory neuropathy and movement disorders. Muscle Nerve 16:904-10.

13. Brooks DJ. A comparison of the abnormal patterns of cerebral activation associated with neuropathic and essential tremor. Neurology 1992;42(Suppl 3):423.

14. Boecker H, Brooks DJ. Functional imaging of tremor. Mov Disord 1998;13:64-72.

15. Herzog J, Hamel W, Wenzelburger R, et al. Kinematic analysis of thalamic versus subthalamic neurostimulation in postural and intention tremor. Brain 2007;130:1608-25. 